\title{
Limited $v s$ full microbiological investigation for the management of symptomatic polymicrobial urinary tract infection in adult spinal cord- injured patients
}

\author{
Rabih O Darouiche, Michael Priebe and Jill E Clarridge \\ Baylor College of Medicine and the Veterans Affairs Medical Center, 2002 Holcombe Boulevard, Houston, Texas \\ 77030, USA
}

\begin{abstract}
Spinal cord-injured (SCI) patients often suffer from symptomatic polymicrobial urinary tract infection (UTI). The objective of this study was to evaluate the clinical outcome and costsavings associated with antibiotic therapy based on limited $v s$ full microbiological investigation of urine cultures in adult SCI patients with symptomatic polymicrobial UTI $(\geqslant 2$ organisms growing in urine cultures). In the first part of the study, a total of 40 evaluable patients were prospectively randomized in a single-blinded fashion to receive antibiotic therapy based on either limited (21 patients) or full microbiologic investigation (19 patients) of urine cultures. The practicality of a limited microbiological investigation was further examined in the second part of the study where 12 consecutive patients with symptomatic polymicrobial UTI initially had only limited microbiological investigation of urine cultures and received antibiotic therapy accordingly. When analyzing all patients in the study, the likelihood of adequate clinical response was not significantly different between those who received antibiotic therapy based on limited $(28 / 33=85 \%)$ vs full $(18 / 19=95 \%)$ microbiological investigation of urine cultures $(P=0.40)$. An average of 183 US dollars could be saved per patient by managing symptomatic polymicrobial UTI based on a limited $v s$ a full microbiological investigation. These results suggest that in adult SCI patients with symptomatic polymicrobial UTI antibiotic therapy guided by a limited microbiological investigation may be practical, adequate and cost-saving.
\end{abstract}

Keywords: microbiological investigation; polymicrobial urinary tract infection; spinal cord injury

\section{Introduction}

Patients with spinal cord injury (SCI) commonly suffer from a neuropathic bladder and become dependent on bladder catheterization. By bypassing the urethra, the most effective defense mechanism against infection, placement of a catheter into the bladder has a high risk of introducing bacteria into the urinary tract. ${ }^{1}$ Although the risk of developing bacteriuria may be lower in SCI patients who undergo intermittent catheterization of the bladder than those with indwelling bladder catheters, urinary tract infection (UTI) represents the most common infection in both groups of patients. ${ }^{2}$ It is not surprising, therefore, that urine specimens constitute the largest number of specimens collected from SCI patients and processed at most clinical microbiology laboratories. ${ }^{3}$

It is generally reported that about $11-31 \%$ of hospitalized patients with symptomatic UTI have polymicrobial growth in urine cultures. ${ }^{4-7}$ An even

Correspondence: RO Darouiche

Presented in part at the 55th Annual Assembly of the American academy of Physical Medicine and Rehabilitation, Miami, Florida, U.S.A., 1993 greater portion of SCI patients with clinical UTI are thought to have polymicrobial urine cultures. For instance, almost half of positive urine cultures from SCI patients with clinical UTI in our institution were observed to grow $\geqslant 2$ organisms in urine cultures; this finding is usually referred to as polymicrobial symptomatic UTI, although not all cultured organisms may contribute to signs and symptoms of UTI. There is no established way to accurately identify which cultured organism(s) is/are responsible for the particular clinical episode of polymicrobial UTI, ${ }^{7}$ thereby impairing the ability to administer antibiotic therapy directed against only the pathogenic organism(s). As a result, physicians generally request that clinical microbiology laboratories identify and perform antibiotic susceptibility on most, if not all, organisms isolated from urine cultures in patients with polymicrobial UTI. This management approach is expensive and labor-intensive and usually leads to the administration of a broad-spectrum antibiotic regimen that can possibly be associated with an increased risk of developing adverse drug effects and bacterial resistance. 
Another potential approach in patients with symptomatic polymicrobial UTI entails administration of antibiotic therapy guided by limited investigation of urine cultures and monitoring the clinical response of patients. Institution of processing controls for investigation of polymicrobial urine cultures at a clinical microbiology laboratory has been reported to reduce the laboratory costs. ${ }^{3,8}$ However, the clinical impact on patient care and the overall savings attributed to guiding antibiotic therapy for symptomatic polymicrobial UTI by only a limited microbiological investigation of urine cultures have not been previously examined, particularly in the population of SCI patients in whom symptomatic UTI is often polymicrobial.

The two main objectives of this study were to: (1) compare the outcome of symptomatic polymicrobial UTI in adult SCI patients who receive antibiotic therapy guided by a limited $v s$ a full microbiological investigation of urine cultures; and (2) determine the cost-savings that result from the use of the former vs the latter approach for managing symptomatic polymicrobial UTI in this population.

\section{Materials and methods}

\section{Patient population}

Male patients who suffered from symptomatic polymicrobial UTI while hospitalized at the 40-bed SCI Unit at the Veterans Affairs Medical Center, Houston, Texas, USA, were eligible for enrollment in this study. An informed consent was obtained for all enrolled patients. Symptomatic polymicrobial UTI was defined by the presence of polymicrobial bacteriuria $(\geqslant 2$ bacterial species growing from urine cultures, each at a concentration of $\geqslant 10^{3} \mathrm{cfu} / \mathrm{ml}$ ) and pyuria $(\geqslant 5 \mathrm{WBC} / \mathrm{HPF})$, in association with one or more of the following clinical manifestations, including fever, flank or suprapubic tenderness, bladder spasm, dysuria, change in voiding habits, nausea or vomiting, increase in spasticity, worsening of dysreflexia, and leukocytosois; other potential etiologies for these clinical manifestations had to be excluded before symptomatic UTI could be diagnosed.

\section{Study design: first part}

This study consisted of two parts. The first part was a prospective, randomized, single-blinded study. Patients enrolled into the first part of the study were randomized into one of two groups (group A or B). Patients in group A received empirical antibiotic therapy guided by the information provided by limited microbiologic investigation of urine cultures, including colony morphology, appearance on Gram-stain, catalase test, and oxidase test (identification of organisms and their antibiotic susceptibilities were not performed). These tests helped identify the group of organisms to which the isolated bacteria belong and, therefore, helped narrow down the selection of empirical antibiotics based on the recognized hospital-based patterns of antibiotic susceptibilities for various groups of organisms. Patients randomized to group B received susceptibility-guided antibiotic therapy based on the information provided by complete microbiological investigation of urine cultures. Identification of bacteria and antibiotic susceptibilities were accomplished by standard bacteriologic methods. ${ }^{9}$ Although a complete microbiological investigation was performed on urine cultures from all patients enrolled in both groups A and B, the results of the complete microbiologic investigation were generally not made available to the physicians who treated patients belonging to group A. Only in instances where patients belonging to group A had not responded to their assigned empirical antibiotic therapy were the results of a complete microbiological investigation made known to the respective physicians. Patients belonging to group A or B were managed by the same group of primary care physicians.

Patients who were clinically judged to be too sick to await the results of the limited or full microbiological investigation of urine cultures were started by the primary care physicians on initial pre-investigation antibiotic therapy sometime after obtaining the urine cultures. When the results of the microbiological investigation became available, patients were managed according to one of three scenarios: (1) freshly started on the assigned form of antibiotic therapy; (2) kept on the same pre-investigation antibiotic regimen that had been initially started (in this case, the day of starting the assigned therapy is considered as that day when initial pre-investigation therapy was started before receiving the results of the microbiological investigation of urine cultures); or (3) switched from the initial pre-investigation antibiotic regimen to the assigned form of therapy (if the initial pre-investigation antibiotic regimen was judged to lack coverage against $\geqslant 1$ of the originally isolated organisms).

Patients with the clinical diagnosis of lower UTI received a one-week course of assigned antibiotics (usually per mouth, except if the results of antibiotic susceptibility testing indicated the need for parenteral agents). Subjects with the clinical diagnosis of pyelonephritis (reflected by the presence of flank pain or tenderness, nausea, vomiting, signs of clinical sepsis, or associated bacteremia) were treated with antibiotics for 2 weeks (usually with parenteral agents during the first week and with oral agents thereafter). Patients were monitored at 3-4 days after initiation of antibiotic therapy and within a day of completing the assigned antibiotic therapy. Monitoring consisted of evaluation of the clinical manifestations of UTI, analysis of pyuria and performance of urine cultures. Adequate response to therapy was defined as clinical improvement within 3-4 days of instituting the assigned antibiotic therapy and which persisted throughout the duration of treatment. Failure to respond to therapy was defined by either: (a) the lack 
of clinical improvement after 3-4 days of receiving assigned antibiotic therapy, or (b) the persistence of some signs and symptoms of UTI at end of treatment, in association with persistent pyuria and bacteriuria due to $\geqslant 1$ of the originally isolated organisms. In order to compare the potential impact of antibiotic therapy guided by limited $v s$ full microbiologic investigation on the recurrence of symptomatic UTI, patients were monitored for recurrence of symptomatic UTI due to $\geqslant 1$ of the originally isolated bacterial species over a period of 1 month after the end of the assigned antibiotic therapy.

\section{Study design: second part}

The second part of the study was designed to examine the practicality of treating a consecutive sample of SCI patients suffering from symptomatic polymicrobial UTI with empirical antibiotic therapy guided by a limited microbiological investigation. Only limited microbiologic investigation was initially performed on urine cultures obtained from patients enrolled into this part of the study, and the bacterial plates were saved at $4{ }^{\circ} \mathrm{C}$; in patients who failed to respond to empirical antibiotic therapy, bacterial plates were retrieved from the refrigerator and the identities and antibiotic susceptibilities of the isolated organisms were determined. Clinical monitoring of patients participating in this part of the study was performed in a fashion similar to that of the first part of the study.

\section{Cost analysis}

The overall costs of managing symptomatic polymicrobial UTI in SCI patients based on a limited $v s$ a full microbiological investigation were assessed by determining: (1) the overall costs of antibiotic therapy, including costs of antibiotic acquisition, preparation, and administration (including personnel time and intravenous sets); and (2) the extra costs charged by a commercial laboratory for conducting a full vs a limited microbiological investigation of the collected polymicrobial urine cultures.

\section{Statistical analysis}

All evaluable patients enrolled into both parts of the study were aggregated for the sake of statistical analysis. Categorical variables were analyzed using either the Chi-square or Fisher's exact test. Continuous variables were compared using the $t$-test. A $P$ value $<0.05$ was considered to be significant. We assumed that finding $>20 \%$ difference in response rates among patients who have only a limited microbiological investigation $v s$ a full microbiological investigation of polymicrobial urine cultures would certainly be clinically important. For instance, using a 2-tailed test and $\alpha=0.05$, the power of this relatively small study for demonstrating a significant difference between an expected response rate of $95 \%$ (this response rate was anticipated from our own experience with this particular patient population) among patients who receive antibiotic therapy based on a full microbiological investigation of polymicrobial urine cultures $v s$ a rate of $65 \%$ among patients who had only a limited microbiological evaluation would have been $81 \%$.

\section{Results}

\section{Clinical characteristics}

A total of 45 SCI patients were enrolled into the first part of the study. Five patients were excluded from analysis because of either co-occurrence of other infections that impaired the ability to clearly evaluate the adequacy of clinical reponse of UTI to the assigned antibiotics (3 patients) or lack of adequate follow-up (two patients). Of the 40 evaluable patients enrolled into the first part of the study, 21 received empirical antibiotic therapy based on a limited microbiological investigation and 19 were treated with antibiotics based on susceptibility patterns. All 12 SCI patients enrolled into the second part of the study were evaluable. The clinical characteristics of all evaluable patients who received empirical antibiotic therapy based on a limited microbiological investigation (a total of 33 patients) and of patients who were treated with antibiotics based on susceptibility patterns (19 patients) were compared in Table 1. The two groups of male patients were well matched as to age, type of bladder drainage, location of UTI, route of administration of antibiotics, initiation of pre-investigation antibiotic therapy before the results of the microbiological investigation became available, isolation of $\geqslant$ multiresistant urinary organism(s), and number of bacterial species isolated from urine cultures.

\section{Response to therapy}

As Table 2 shows, it took a significantly shorter period of time after obtaining urine cultures to start the assigned antibiotic therapy based on a limited $v s$ a full microbiological investigation of urine cultures (mean $\pm \mathrm{SD}$ of $1.2 \pm 1.4$ days $v s \quad 3.3 \pm 2.5$ days, respectively; $P=0.01)$. Among those who had initially received pre-investigation antibiotic therapy prior to obtaining results of urine cultures, a significantly higher number of patients who had a limited $v s$ a full microbiological investigation of urine cultures required no change in antibiotic therapy after obtaining culture results $(85 \%$ vs $33 \%$, respectively; $P=0.006)$. Clinical response was not significantly different between those who received antibiotic therapy guided by limited $v s$ full microbiological investigation $(85 \%$ vs $95 \%$; $P=0.40)$.

All five patients (three enrolled into the first part of the study and two enrolled into the second part of the study) who had failed to clinically improve after 3-4 days of receiving the assigned antibiotic therapy for UTI (one case had pyelonephritis) based on a limited 
Table 1 Characteristics of 52 evaluable patients with symptomatic polymicrobial UTI

\begin{tabular}{|c|c|c|c|}
\hline Characteristic & $\begin{array}{l}\text { Empirical therapy } \\
\quad(n=33)\end{array}$ & $\begin{array}{l}\text { Susceptibility-based therapy } \\
(n=19)\end{array}$ & $\mathrm{P}$ value \\
\hline Mean age in years \pm SD (range) & $47.5 \pm 12.0(23-84)$ & $52.7 \pm 12.0(23-69)$ & N.S. \\
\hline \multicolumn{4}{|l|}{$\begin{array}{l}\text { No. }(\%) \text { with particular type of bladder } \\
\text { drainage }\end{array}$} \\
\hline Indwelling catheter & $17(52)$ & $13(68)$ & N.S. \\
\hline Intermittent catheter & $8(24)$ & $4(21)$ & N.S. \\
\hline Ileal conduit & $4(12)$ & $0(0)$ & N.S. \\
\hline External condom & $4(12)$ & $2(11)$ & N.S. \\
\hline No. $(\%)$ with pyelonephritis & $6(18)$ & $5(26)$ & N.S. \\
\hline No. (\%) who received IV antibiotics & $16(48)$ & $14(74)$ & N.S. \\
\hline $\begin{array}{l}\text { No. }(\%) \text { who received initial empirical } \\
\text { therapy before investigating cultures }\end{array}$ & $20(61)$ & $12(63)$ & N.S. \\
\hline No. (\%) with multiresistant organisms & $22(67)$ & $12(63)$ & N.S. \\
\hline $\begin{array}{l}\text { Mean no. of bacterial species in urine } \\
\text { cultures } \pm S D \text { (range) }\end{array}$ & $2.8 \pm 0.8(2-5)$ & $2.7 \pm 0.8(2-4)$ & N.S. \\
\hline
\end{tabular}

$\mathrm{UTI}=$ urinary tract infection; N.S. $=$ not significant

Table 2 Comparison of findings in 52 evaluable patients treated for symptomatic polymicrobial UTI

\begin{tabular}{|c|c|c|c|}
\hline Finding & $\begin{array}{l}\text { Empirical therapy } \\
(n=33)\end{array}$ & $\begin{array}{l}\text { Susceptibility-based therapy } \\
(n=19)\end{array}$ & $\mathrm{P}$ value \\
\hline $\begin{array}{l}\text { Mean no. of days from urine culture to } \\
\text { starting assigned therapy (range)* }\end{array}$ & $1.2 \pm 1.4(0-4)$ & $3.3 \pm 2.5(0-8)$ & 0.01 \\
\hline $\begin{array}{l}\text { No. kept on initial pre-investigation therapy/ } \\
\text { total no. received initial pre-investigation } \\
\text { therapy }(\%)\end{array}$ & $17 / 20$ & $4 / 12$ & 0.006 \\
\hline $\begin{array}{l}\text { No. }(\%) \text { with adequate response to assigned } \\
\text { antibiotic therapy }\end{array}$ & $18(85)$ & $18(95)$ & 0.40 \\
\hline
\end{tabular}

UTI = urinary tract infection. * Refers to all patients, including those who either received or did not receive initial preinvestigation antibiotic therapy before obtaining results of investigations of urine cultures

microbiological investigation responded adequately when switched to susceptibility-based antibiotic therapy without any residual complications of UTI. The organisms that had not been covered by the originally assigned antibiotic regimens and, therefore, were thought to have been associated with inadequate clinical response included Citrobacter freundii (at a concentration $>10^{5} \mathrm{cfu} / \mathrm{ml}$ ) in one case, Enterobacter aerogenes (at a concentration $>10^{5} \mathrm{cfu} / \mathrm{ml}$ ) in one case, Providencia rettgeri (at a concentration $>10^{4}$ but $<10^{5} \mathrm{cfu} / \mathrm{ml}$ ) in one case, a combination of Escherichia coli and Staphylococcus aureus (both at a concentration $>10^{5} \mathrm{cfu} / \mathrm{ml}$ ) in one case, and a combination of $S$. aureus, Enterococcus faecalis and group B Streptococcus species (all at a concentration $\geqslant 10^{5} \mathrm{cfu} / \mathrm{ml}$ ) in the fifth case. The only patient who failed to respond to the assigned susceptibility-based antibiotic therapy had a persistent infection by Providencia stuartii (at a concentration $>10^{5} \mathrm{cfu} / \mathrm{ml}$ ) that became resistant to the assigned antibiotic (gentamicin); selection of another susceptibility-based antibiotic (cefotaxime) eventually cleared this symptomatic infection.
Urine cultures obtained at the end of therapy grew yeast in $3 / 33(9 \%)$ patients who were treated based on a limited microbiological investigation vs 3/19 (16\%) patients who received susceptibility-based antibiotic therapy $(P=0.66)$. Recurrence of symptomatic UTI due to $\geqslant 1$ of the originally infecting bacterial species within a month of discontinuing the assigned antibiotic therapy was equally observed in the two groups of patients $(8 / 33=24 \%$ vs $4 / 19=21 \%$, respectively; $P=1.0$ ).

\section{Cost comparison}

The mean overall cost of antibiotic therapy tended to be lower in patients in whom selection of antibiotic therapy was based on a limited $(157 \pm 174$; range, $0.1-560$ US dollars) vs full (252 \pm 237 ; range, $0.1-882$ US dollars) microbiological investigation of urine cultures; the difference (mean, 95 US dollars), however, was not statistically significant $(P=0.18)$. The mean extra amount of money charged by a commercial laboratory to perform a full vs a limited microbiological investigation was 88 US dollars for each polymicrobial urine culture. 


\section{Discussion}

The findings of this study suggest that empirical antibiotic therapy guided by a limited microbiological investigation of urine cultures may be as effective as susceptibility-based antibiotic therapy for symptomatic polymicrobial UTI in male SCI patients. In the rare occasions (15\% of cases) when such an approach failed to produce an adequate clinical response, further evaluation of organisms isolated from the agar plates that had been saved in the refrigerator provided the necessary antibiograms for initiation of susceptibilitybased antibiotic therapy that was regularly curative without any residual complications of UTI. Failure to respond to empirical therapy guided by a limited microbiological investigation of polymicrobial urine cultures could have been caused by the lack of antibiotic coverage for $\geqslant 1$ implicated urinary pathogens. In agreement with previous reports, ${ }^{7,10-12}$ neither the type (both Gram-positive and -negative bacteria were implicated pathogens) nor the concentration (some pathogenic bacteria were present at concentrations $<10^{5} \mathrm{cfu} / \mathrm{ml}$ ) of organisms in urine could help differentiate in this study between pathogenic and colonizing bacteria. The findings of similar occurrences of fungal superinfection and UTI relapse in patients who received antibiotic therapy guided by limited vs full microbiological investigation of urine cultures may yield further support to the adequacy of the former management approach.

In addition to possibly providing an adequate clinical response in SCI patients with symptomatic polymicrobial UTI, the findings of this study suggest that empirical antibiotic therapy guided by a limited microbiological investigation of urine cultures can also be practical and cost-saving. This approach was associated with a quicker initiation of appropriate therapy and a lower likelihood for changing the initially instituted pre-investigation antibiotic regimen in patients who required treatment before investigating urine cultures. In other words, the institution of such an approach may facilitate the management and speed-up the appropriate disposition of patients. Although differences were not statistically significant, patients who received antibiotic therapy based on a full microbiological investigation were more likely to receive intravenous antibiotics. These differences could not have been caused by differences in the frequency of isolation of multiresistant urinary pathogens between the two groups of patients, but could have been attributed to the fact that administration of susceptibility-guided antibiotic therapy necessitated the use of intravenous antibiotics even in patients who could have tolerated oral therapy. Mean total savings of 183 US dollars per patient, equivalent to the sum of the extra laboratory charges for a full vs a limited microbiological investigation of polymicrobial urine cultures (mean, 88 US dollars) and the difference in the overall costs of antibiotic therapy in patients whose therapy is guided by a full $v s$ a limited microbiological investigation (mean, 95 US dollars), could be anticipated from using this management approach.

In subjects with a functional bladder urine cultures that yield polymicrobial growth are usually regarded as contaminated specimens and discarded, and repeat urine cultures are frequently requested. However, polymicrobial urine cultures can sometimes represent a true infection as demonstrated by the simultaneous growth of $\geqslant 1$ identical organisms in blood and urine cultures from patients with urosepsis ${ }^{13}$ or by the repeated growth of the same mixture of organisms in multiple urine specimens. ${ }^{7}$ Our findings support the notion that polymicrobial growth in urine cultures obtained from symptomatic SCI patients can be clinically meaningful. The results of this study may apply to other types of patients who are dependent on bladder catheterization, such as stroke victims, elderly subjects, and nursing home residents.

\section{Conclusions}

The findings of this study suggest that the treatment of symptomatic polymicrobial UTI in adult SCI patients with empirical antibiotic therapy guided initially by a limited microbiological investigation may be adequate, practical, and cost-saving. Knowledge of the hospitalbased patterns of antibiotic susceptibilities for various groups of organisms is essential for the success of this approach. Bacterial plates can be initially saved in the refrigerator and, in patients who fail to improve after 3-4 days of such antibiotic therapy, retrieved later for identification and antibiograms of all isolated organisms. The utility of this management approach merits further examination in larger-sized clinical trials in SCI patients as well as in other subjects who require continuous bladder drainage.

\section{References}

1 Donovan WH, Stolov WC, Clowers DE, Clowers MR. Bacteriuria during intermittent catheterization following spinal cord injury. Arch Phys Med Rehabil 1978; 59: 351-357.

2 Warren JW, Muncie HL, Berquist EJ, Hoopes JM. Sequelae and management of urinary infections in the patient requiring chronic catheterization. J Urol 1981; 125: 1 -8.

3 Bartlett RC, Treiber N. Clinical significance of mixed bacterial cultures of urine. Am J Clin Pathol 1984; 82: 319-322.

4 Platt R, Polk BF, Murdock B, Rosner B. Mortality associated with nosocomial urinary tract infection. $N$ Engl J Med 1982; 307: $637-642$.

5 Asher EF, Oliver BG, Fry DR. Urinary tract infections in the surgical patient. Am Surgeon 1988; 54: 466-469.

6 Gebhart RJ, Duma RJ, Patterson PM. Timentin in the treatment of symptomatic urinary tract infection in adult patients. $A m J$ Med 1985; 79: $101-105$.

7 Siegman-Igra Y, Kulka T, Schwartz D, Konforti N. The significance of polymicrobial growth in urine: contamination or true infection. Scand J Infect Dis 1989; 25: 85-91.

8 Bartlett RC, Rutz C. Processing control and cost in bacteriology. Am J Clin Pathol 1980; 74: 287-296. 
9 Baron EJ, Weissfeld AS, Fuselier PA, Brenner DJ. Classification and identification of bacteria. In: Murray PR, Baron EJ, Pfaller MA, Tenover FC, Yolken RH (ed). Manual of Clinical Microbiology. Sixth Edition. American Society for Microbiology: Washington, 1995, pp 249-264.

10 Roberts FJ. Quantitative urine culture in patients with urinary tract infection and bacteremia. Am J Clin Pathol 1986; 85: $616-$ 618 .
11 Strand CL, Bryant JK, Sutton KH. Speticemia secondary to urinary tract infection with colony counts less than $10^{5} \mathrm{CFU} / \mathrm{ml}$. Am J Clin Pathol 1985; 83: 619-622.

12 Stark RP, Maki DG. Bacteriuria in the catheterized patient. $N$ Engl J Med 1984; 311: 560-564.

13 Gross PA, Flower M, Barden G. Polymicrobic bacteriuria: significant association with bacteremia. J Clin Microbiol 1976; 3: $246-250$ 